\title{
Block-based Motion Estimation in Video Frames using Artificial Neural Networks: A Selective Review
}

\author{
Krishna Kumar \\ Department of Computer \\ Application \\ IFTM University, Moradabad, \\ Uttar Pradesh, India
}

\author{
Krishan Kumar, PhD \\ Department of Computer \\ Science \\ Faculty of Technology, \\ Gurukul Kangri University, \\ Haridwar, Uttarakhand, India
}

\author{
Rahul Mishra, PhD \\ Department of Computer \\ Application \\ IFTM University, \\ Moradabad, Uttar Pradesh, \\ India
}

\begin{abstract}
Nowadays, we are very frequently transmitting the video over internet. This is due to an extensive increase in multimedia applications over hand held devices, such as smart mobile phones and also other advance conventional devices. Motion Estimation is an important field of study in the area of motion analysis and motion compression. The motion estimation is done by using two basic approaches, namely, pixel-based motion estimation and block-based motion estimation. Here we have proposed a detailed study literature survey and review of the block-based estimation methods in detail. This paper presents a comprehensive review of block based motion estimation techniques which plays a vital role in multimedia transmission over public network. The advantage of this review paper is to find the absolute optimal solution.
\end{abstract}

\section{General Terms}

Motion estimation, Block Based Motion, Neural networks.

\section{Keywords}

Motion estimation; video compression; motion Vectors.

\section{INTRODUCTION}

In the present time multimedia transmission of good quality is very massive task over the public network. The technology has been improved upto a good extent with the availability of best quality of coding, compression tools and methods, and by the acceptance of international standards proposed by ISO Moving Picture Experts Group (MPEG) video coding standards that deal with compressed video storage and the International Telecommunications Union (ITU) standard that deal with real time point-to-point or multipoint communication [1-3]. There are two techniques to major motion estimation i.e. first one is Pixel-based motion estimation and second one is Block-based motion estimation.

\subsection{Pixel-Based Motion Estimation}

This method explores two major techniques for finding the relationship between two frames, namely, first is frame replenishment and second is motion compensation. The frame replenishment technique is conditional for only those pixel gray level values whose variation from their counterparts in the previous frame exceeds a threshold. There are only encoded and transmitted to the receiver. This simple frame replenishment technique has higher coding efficiency in comparison with the coding scheme where each pixel in each frame is coded. There exist other advance frame replenishment techniques such as frame difference prediction coding technique etc.

The frame replenishment techniques do not work effectively when there is a rapid motion and/or intensity variation occurring on the image planes. In such situation, it is to rely on motion compensated coding in which the motion of pixel in a frame is first analyzed. Now, based on the previous frame and the estimated motion, the current frame is predicted. It has, however, been noted that the right coding efficiency is obtained in motion compensation at the cost of higher computational complexity [5-7].

\subsection{Block-Based Motion Estimation}

Block-based motion estimation approach which is more popular and is widely used in video coding standards such as ITU H.261, H.263, H.264, MPEG-1, MPEG-2 and MPEG-4. In this technique, non-overlapping blocks of pixels, known as macro blocks, are usually predicted from previous frames. This motion information is represented by displacement vectors or motion vectors. The motion vector minimizes a cost function involving the candidate and the target macro blocks. There are a number of cost functions that can be used. The most widely used function is the Sum of Absolute Difference $(S A D)$. The macro block producing the minimum $S A D$ is computed within a given search area. The block-based motion estimation methods rely on the algorithm called the BlockMatching Algorithms (BMAs). BMAs are popular because of their simplicity of concepts and implementation. One of the simplest $B M A$ which is known as the full search $B M A$, calculate the $S A D$ in the search area at every possible pixel location. This procedure usually consumes more time. Hence, the requirement arises for the development of fast blockmatching methods to reduce the search time. A number of methods have been proposed in literature in order to increase the efficiency of the process of block-matching. These methods generally focus on reducing the computational time and on increasing the quality of image.

Cryptography used from accent time for secure communications. In cryptographic algorithm mathematical functions are used for encryption and decryption process as traditional methods. The basic terms used in Motion Estimation and Motion Compensation.

\subsection{Motion Estimation and Motion Compensation}

Motion estimation is based on the temporal prediction technique. The basic idea behind the motion estimation is to compute the displacement between the current frame and the previous frame. The previous frame is also referred as the reference frame. The video coding standards, such as the H.264 present the flexibility in selecting the reference frames and their combinations can also be selected. It considers a pixel belonging to the current frame in organization with its region as that of the candidate and then determines its best matching position in the reference frame. The difference in position between the candidates and its match in the reference 
frame is defined as the displacement vector or motion vector. While determining the motion vectors, the current frame can be predicted by applying the displacements corresponding to the motion vectors on the reference frame. This method is termed as motion compensation.

During the transmission of video, for the removal of interframe redundancy, reference frame and the motion vector information need to be coded. Each pixel is propagated along its motion trajectory to reproduce the pictures. During this in order to reduce transmitting motion vector information cost, assumed that the pixels in the same group are have the same motion information. This assumption always not true because the pixels in the block may move in different direction or some of them may belong to the background. Therefore, both motion vectors and the prediction difference have to be transmitted. Because of less computational complexity, the block matching can be considered as the most practical method for motion estimation. The image frame is divided into fixed-size small rectangular blocks such as $16 \times 16$ or $16 \times 8$ or $4 \times 4$ in video coding in the block-matching method. Each block assumed to undergo a linear translation and the displacement vector of each block and the predictive errors are coded and transmitted [8].

\subsection{Block Matching}

As claimed earlier, block-matching is the most popular method being used for motion estimation because of its simplicity and reasonable performance. Block-Matching aims to find the motion of objects in two frames of image by blockbased comparisons. The images are divided into blocks of pixels of equal size. For each block in the current frame, the best matching block in the reference frame is searched. Normally, the block minimizing the estimation error is selected as the matching block.

As the name indicates in the Block-Matching method, we estimate the motion vector on the block-by-block basis. A frame is divided into $(N \times N)$ pixels size blocks. The block (also called as current block) of pixels in the current frame is compared with the blocks within a search area, say, $(N \times 2 p)$ $\times(N \times 2 p)$ pixels in the previous frame. The blocks in the previous frame are called candidate blocks. It is worth mentioning that $p$ is the maximum displacement that is allowed. This concept of the block-matching is described in "Fig.1".

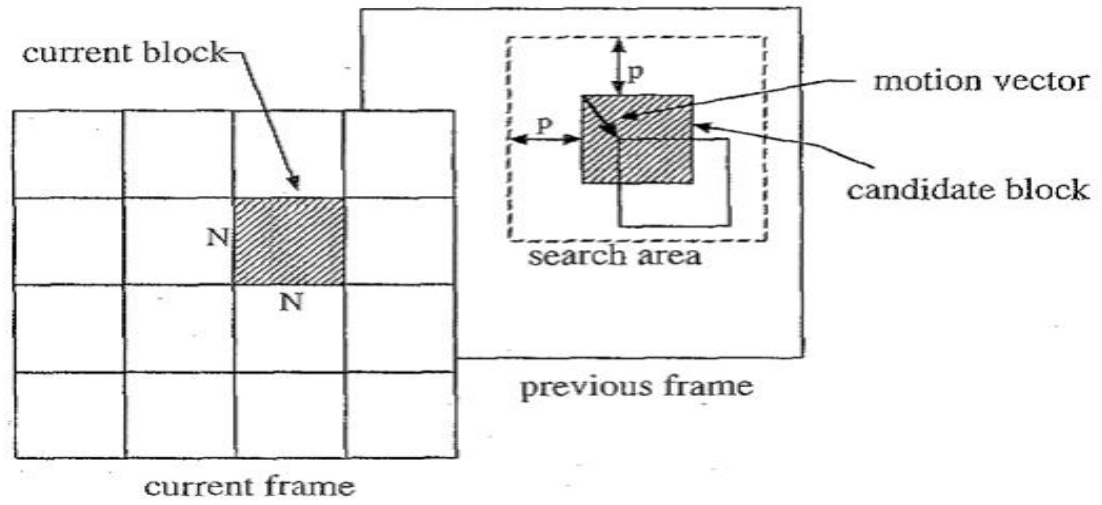

Fig1: Basic of concept of the block-matching process

Let us assume that $R(i, j)$, i.e., reference point is the 1

Let us assume that $R(i, j)$, i.e., reference point is the location of uppermost left pixel of the current block in the current frame and $S(i+u, j+v)$ is the search point, the location of the pixel on the candidate block in the previous frame. Thus the previous frame is shifted by $u$ horizontal pixel and $v$ vertical pixels within the search area. Thus, $(u, v)$ represents the estimate of the displacement in horizontal and vertical directions. The accuracy of the estimation of $(u, v)$ depends upon the methods that are applied for matching. Usually there are five popular matching methods which are described one by one in the subsequent paragraphs.

\subsubsection{Normalized Cross-Correlation Function (NCCF)}

The similarity measure or correlation measure is a key element in the matching process. The basic correlation between the two frames, i.e., current frame and previous frame, is defined as follows [9].

$$
\operatorname{NCCF}(u, v)=\frac{\sum_{i=1}^{N} \sum_{j=1}^{N} R(i, j) \cdot S(i+u, j+v)}{\left[\sum_{i=1}^{N} \sum_{j=1}^{N} R^{2}(i, j)\right]^{\frac{1}{2}}\left[\sum_{i=1}^{N} \sum_{j=1}^{N} S^{2}(i+u, j+v)\right.}
$$

In this computation, the smallest $\operatorname{NCCF}(u, v)$ within the search represents the best match.

\subsubsection{Mean Squared Difference (MSD)}

MSD is commonly called as Mean Square Error (MSE). In this criterion, the MSE is computed to find out the minimum value of MSE and this is computed for each displacement position $(\mathrm{u}, \mathrm{v})$ within a specified search range in the previous frame.

$$
\operatorname{MSD}(u, v)=\frac{1}{N^{2}} \sum_{i=1}^{N} \sum_{j=1}^{N}[R(i, j)-S(i+u, j+v)]^{2}
$$

\subsubsection{Mean Absolute Difference (MAD)}

In this measure, the smallest $M A D(u, v)$ used to determine the motion vector for all possible displacement $(u, v)$ within the search area. Because of its lower computational complexity the $M A D$ is most widely used for matching criterion [10].

$$
M A D(u, v)=\frac{1}{N^{2}} \sum_{i=1}^{N} \sum_{j=1}^{N}|R(i, j)-S(i+u, j+v)|^{\mid}
$$




\section{d) The Peak-Signal-to-Noise-Ratio (PSNR)}

This is the most commonly used as a measure of quality of reconstruction of image compression. Original data is the signal in this case and error is the noise engaged by compression. Compression codecs are used as an approximation to human perception of reconstruction quality. A higher PSNR would normally indicate the higher quality of reconstruction [11]. PSNR characterizes the motion compensated image that is created by using macroblocks and motion vectors from the reference frame. The equation for the PSNR is as given below.

$$
\begin{aligned}
& \text { PSNR } \\
& =10 \log _{10}\left[\frac{(\text { Peak to peak value of original data })^{2}}{M S E}\right]
\end{aligned}
$$

We ask that authors follow some simple guidelines. In essence, we ask you to make your paper look exactly like this document. The easiest way to do this is simply to download the template, and replace the content with your own material.

\section{OVERVIEW OF NEURAL NETWORK}

Mc Culloch \& Pitts were the first persons who introduced artificial neural networks in 1943 . They spent nearly 20 years research to find out that how our brain thinks. An artificial neural network or ANN is inspired from human biological brain and nervous system. Basically our brain is the combination millions of small processing units called neurons. These neurons are means of transferring information in entire nervous system. A neuron just similar to biological cells which transfers information in the electrical form of electro chemical signals, from one neuron to another neuron. Another two important terms i.e. dendrite that receives the information in and axon which transfers information away. They form a tree like structure. The cell body is called soma. Synapse connects the two neurons and also the signals are summed at this junction point "Fig.2".

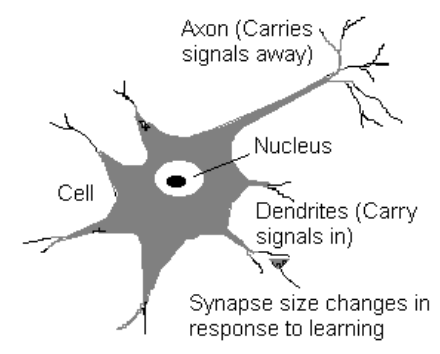

Fig2. Biological Neuron

ANN is the combination of units, connections and weights. The primary architecture of ANN is given in "Fig.3".

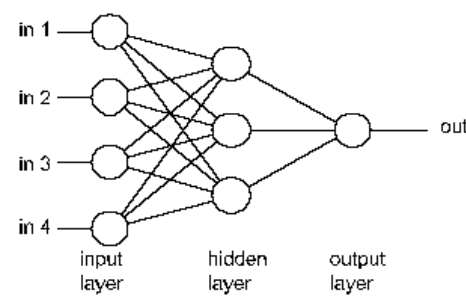

Fig3. Artificial Neural Network
The Comparison symmetric between the basic biological neuron and present modern artificial neuron network:

Table1. Comparison of Biological \& Artificial Neuron

\begin{tabular}{|l|l|}
\hline Biologic NN & Artificial NN \\
\hline Soma & Unit \\
axon, dendrite & Connection \\
synapse & weight \\
potential & weighted sum \\
threshold & bias weight \\
signal & activation \\
\hline
\end{tabular}

It is being widely used in many application areas of research successfully. In the case of the block matching, neural networks work well as a type of learning algorithm, which have been shown to be very useful to improve many aspects of the frame based block- matching and motion estimation [28], [29].

\section{LITERATURE REVIEW}

In this section, the literature on various topics considered in this proposal is being discussed. The topics covered in review of literature include frame based block- matching, object based block-matching, search algorithms for block-matching, and motion estimation using ANN.

Jain and Jain [12] proposed Fixed Size Block-Matching (FSBM) technique. Each frame is divided into a predefine number of square blocks in this technique. In the reference frame, a search is done for each block over a particular part of the image that helps the coder to use for maximum translation. It is for the best matching block to give the least prediction error, usually minimizing either mean square difference or mean absolute difference, which is easier to compute.

Kilthau et al. [13] discussed a novel data structure that uses the Fast Fourier Transform (FFT) in its computation of the Sum Squared Difference (SSD) metric. The use of SSD metric allows higher Peak Signal to Noise Ratio (PSNR) rather than FSBM technique which requires the sum of absolute difference (SAD) metric. It evaluates the cost function in the search window at each possible location. Fast block-matching algorithms try to achieve the same PSNR, doing as little computation as possible. It is always a necessity to choose a block-size to use for motion compensation. A number of smaller blocks represent complex motion better than fewer large ones. This help out to reduces the costs of work and transmission in subsequent correction stages but not deal with the quality for the motion information.

Chan et al. [14] discussed the Variable Size Block-Matching (VSBM) motion compensation scheme based on blockmatching, where the size for each block is a variable. This method divides the image into blocks of variable sizes to get the uniform motion for all blocks according to the assumption. This scheme successfully implemented on the simple interface video coding. The most important of this technique is an improvement in FSBM by varying the size of blocks to more accurately match moving areas.

Packwood et al. [15] proposed object based block-matching motion compensation technique. In this technique, the objectbased video coding standards permit arbitrary-shaped objects to be encoded and decoded as separate Video Object Planes (VOPs). This allows the user to change the composition of the scene at the decoder, and to control other functions such as 
spatial and temporal scaling to maximize the best use of the available bandwidth.

Ma et al. [16] proposed an efficient rate-control algorithm for H.264/AVC video encoding. A result in the relationship between the quantization parameter and the true quantization step size is quantization scheme in H.264/AVC. Which is no longer linear. A new Rate-Distortion (R-D) model by utilizing the true quantization step size is proposed and then developed an improved rate-control scheme for the H.264/AVC encoder based on R-D model. In general, the current R-D Optimization (RDO) mode-selection scheme in H.264/AVC test model is difficult for rate control, because rate control usually requires a predetermined set of motion vectors and coding modes to select the quantization parameter, whereas the RDO does in the different order and requires a predetermined quantization parameter to select motion vectors and coding modes.

\section{ALGORITHM FOR BLOCK MATCHING}

The searching strategy is an important issue to deal with in block-matching. For searching the best match, the correlation window is moved to each candidate's position within the search window, i.e., the total area of the frame for block position is needed to be examined. The minimum dissimilarity gives the best match. Several fast searching procedures proposed by different researchers are given here in this subsection.

Koga et al. [17] proposed Three-Step Search (TSS) technique in which the best motion vectors are searched out to find search patterns. This is different from the algorithm proposed by Jain and Jain in two significant respects: the use of Mean Absolute Distortion (MAD) instead of Mean Square Error (MSE), and a somewhat different search pattern. The general idea is represented in "Fig.2", where it finds the location of the best match with the least cost function and the macro block at that location. The calculated motion vector is then saved for transmission.

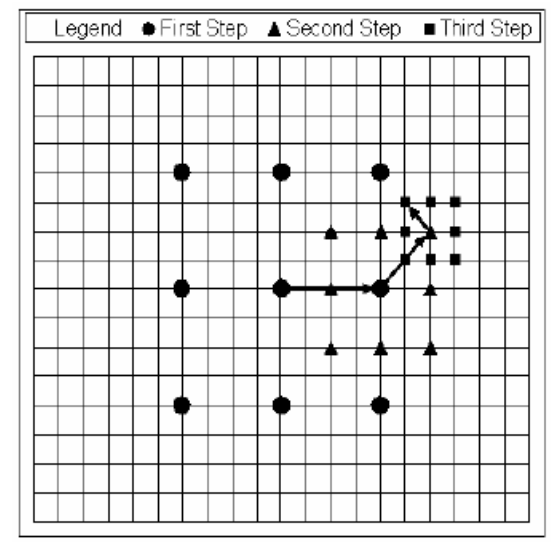

Fig4. Three Step Search Procedure

Renxiang et al. [18] proposed New Three Step Search (NTSS) algorithm, which is an improvement on TSS results by providing a center biased searching scheme and having provisions for half way stop to reduce computational cost. Like MPEG-1 and H.261 it was one of the first widely accepted fast algorithms and frequently. The TSS uses a uniformly allocated checking pattern for motion detection and is prone to missing small motions. It has the same steps as the TSS, adding points neighboring the center point of block to the checked block in the first step.
Lu and Liou [19] discussed the Simple and Efficient Search (SES) algorithm. This is another extension to TSS and exploits the assumption of unmoral error surface. The main idea behind the algorithm is the search pattern in TSS, at most half of the total eight directions need actually be searched in each step, and thus, the computational

complexity can be further reduced. On the other hand, to determine which directions are to be chosen, additional computation is needed.

$P o$ and Ma [20] proposed the Four Step Search (4SS) algorithm. In this algorithm, center-biased checking point pattern for real world image sequence fast block motion estimation has been discussed. In the new algorithm with searching steps of 2 to 4 and the total number of checking points is varied from 17 to 27 and Halfway-stop technique is employed. This has similar performance to NTSS in terms of motion compensation errors and gives better performance than the well-known three-step search algorithm. This algorithm reduces the average computational requirement from 21 to 19 search points and computational requirement from 33 to 27 search points as compared with NTSS.

Zhu and Ma [21] proposed Diamond Search (DS) algorithm. The implementation of this algorithm is based on $4 S S$, by changing the square search to a diamond search. $D S$ uses two different types of fixed patterns, one is Large Diamond Search Pattern (LDSP), comprising nine checking points from which eight points surround the center to compose a diamond shape and the other is Small Diamond Search Pattern (SDSP) consisting of five checking points which forms a smaller diamond shape. LDSP is repeatedly used in the searching method of the $D S$ algorithm until the step in which the Minimum Block Distortion $(M B D)$ occurs at the center point. The search pattern is then switched from LDSP to SDSP as reaching to the final stage. Among the five checking points in SDSP, the position yielding the MBD provides the motion vector of the best matching block.

Nie and Ma [22] studied Adaptive Rood Pattern Search (ARPS) method. The ARPS size is dynamically determined for each macroblock, based on the available motion vectors (MVs) of the neighboring MBs. ARPS method adaptively exploits adjustable rood-shaped search pattern, together with the search point indicated by the predicted MV, to match different motion contents of video sequence for each macroblock. The Zero-Motion Prejudgment (ZMP) was incorporated into ARPS to further benefit small motion video sequence. In their study, they compared DS with ARPS-ZMP, and observed that the computational gain significantly increases the factors ranging from 1.9 to 3.4 with little reduction in average PSNR. In addition, ARPS-ZMP improves average PSNR performance in large motion video sequences.

\section{MOTION ESTIMATION USING ARTIFICIAL NEURAL NETWORK}

Slanina and Ricny [23] designed a network to estimate PSNRs of the video sequence frames, which enabled computation of full reference objective quality metric values without having the undistorted video material prior to encoding for comparison. It was designed for both, using low resolution video sequences, and with increased resolution also, it performed well.

Flevry and Egger [24] designed a way to predict the coding quality of a region of the input image for block-based coding method. The system was able to select the best suited coding 
algorithm for each region according to the predicted quality. The prediction system was built using the artificial neural networks.

Zhang and Zhang [25] proposed a prediction method based on the Radial Basis Function (RBF) neural network to realize prediction for every single encoding quantization parameter fast and effective. This method trained the neural network by selecting part continuous frame video frequency in the video sequence, concluded that predictive value to quantization parameter using the well-trained neural network fastly, depends upon the neural network's fault-tolerant ability to strengthen self-adaption of the system, to make accurate prediction for image.

Malhotra and Takaya [26] proposed artificial neural network based method to detect moving object in video sequence by performing image segmentation on the frame sequences based on the criteria of motion and then applied the motion vector estimation algorithm to find the geometrically identical points in two consecutive video frames.

Gersho and Gray [27] studied quantization technique which capitalizes any underlying structure in the data being quantized. The space of the vectors to be quantized is divided into a number of regions. A reproduction vector is calculated for each region. Given any data vector to be quantized, the region in which it lied was determined and the vector was represented by the reproduction vector for that region.

Berkane and Clarysse [28] developed a method for motion estimation in image sequences based on neural network architecture. This network had a specific topology dedicated to motion estimation inspired from the human vision system. It combined the advantages of both the block-matching method and neural networks.

Ryu Dae-Hyun [29] proposed a method that predicts the search region by Vector Quantization (VQ) using neural network for the motion estimation. It reduces the computation because it uses smaller number of search points than conventional methods and reduces the bit required to represent motion vectors. In these studies, the MV is taken by using full search BMA from the initial image sequences. The trained Frequency Sensitive Competitive Learning (FSCL) neural networks were designed using the motion vectors and called as codebook. This codebook is used as motion estimation region. It used the spatial correlation of motion vectors in image sequences, therefore reduces the search area, bits required to transmit motion vectors and increased the compression rate. This method is superior to the TSS and robust to the noise because it has the motion vector which has smoothing effect during the vector quantization process.

\section{CONCLUSION}

Motion Estimation is the essential process in video compression and primary reason for computational complexity of video compression. The prime objective of this research paper is to survey the optimal solutions of the different video compression problems. In this paper, a review of various algorithms, such as Three-Step Search technique, Simple and Efficient Search algorithm, Four Step Search algorithm, Diamond Search algorithm have been presented and discussed one by one. Consequently it is found that artificial neural network may play a very important role in motion estimation issue. Moreover from the analysis, It is observed that use of artificial neural networks in motion estimation techniques produces better quality image as it gives better performance in PSNR calculation. The image quality can be improved by increasing the PSNR values with the use of neural networks. Therefore, we can say that the goal of achieving better quality images in motion estimation using neural networks is desirable.

\section{REFERENCES}

[1] Emmanuel Reusens, Touradj Ebrahimi and Murat Kunt, "Dynamic coding of visual information", IEEE Transactions on Circuit and Systems for Video Technology, vol. 7, no. 3, 1997, pp. 489-500.

[2] Detlev Marpe, Thomas Wiegand and Gary J. Sullivan, "The H.264/MPEG4 advanced video coding standard and its applications", IEEE Communications Magazine, 2006, pp. 134-143.

[3] Karel Rijkse and KPN Research, "H.263: Video coding for low bit rate communication", IEEE Cominunications Magazine, 1996, pp. 42-45.

[4] Thomas Sikora, "The MPEG-4 video standard verification model", IEEE Transactions on Circuit and Systems for Video Technology, vol. 7, no. 1, 1997, pp. 19-31.

[5] Borko Furht, Joshua Greenberg and Raymond Westwater, "Motion estimation algorithm for video compression", Kluwer Academic Publishers, Norwell, MA, 1997, Ch. 2 and 3.

[6] Iain E.G. Richardson, "Video Codec Design", John Wiley and Sons Ltd., 2002, Ch. 4, 5 and 6.

[7] J. Ribas-Corbera and D.L. Neuhoff, "On the optimal block size for block-based motion compensated video coders", SPIE Proceedings of Visual Communications and Image Processing, vol. 3024, 1997, pp. 1132-1143.

[8] Y. Wang and Q. Zhu, "Error control and concealment for video communication: a review", Proceedings of the IEEE, special issue on Multimedia Signal Processing. vol. 86, 1998, pp. 974-997.

[9] H. G. Musmann, P. Pirsch and H. J. Grallert, "Advances in picture coding", IEEE Proceeding of video coding, vol. 73 , no. 4 , 1985 , pp. 523-548.

[10] M. J. Chen, L. G. Chen, T. D. Chiues, and Y. P. Lee, "A new block matching criterion for motion estimation and its implementation", IEEE Transaction on Circuits System Video Technology, vol. 5, no. 6, 1995, pp. 231236.

[11] Huynh-Thu and Ghanbari, M., "Scope of validity of PSNR in image/video quality assessment", Electronics Letters, vol. 44, no. 13, 2008, pp. 800-801.

[12] J.R. Jain and A.K. Jain, "Displacement measurement and its application in interframe image coding", IEEE Transaction on Communication, vol. COM-29, no. 12, 1981, pp. 1799-1808.

[13] Steven L. Kilthau, Mark S. Drew and Torsten Moller, "Full search content independent block matching based on Fast Forier Transformation", In Proceeding of the IEEE conference on ICIP, 2002, pp. 669-672.

[14] M.H. Chan, Y.B. Yu and A.G. Constantinides, "Variable size block matching motion compensation with applications to video coding", IEE Proceedings of advance in video coding, vol. 137 , no. 4 , 1990, pp. 205 212. 
[15] R.A. Packwood, M.K. Steliaros and G.R. Martin, "Variable size block matching motion compensation for object-based video coding", IEEE 6th International Conference on Image Processing and its Applications, Dublin, Ireland, 1997, pp. 56-60.

[16] Siwei Ma, Wen Gao and Yan Lu, "Rate-Distortion analysis for H.264/AVC video coding and its application to rate control", IEEE Transaction on Circuits and Systesm for Video Technology, vol. 15, no. 12, 2005, pp. $1533-1544$

[17] T. Koga, K. Iinuma, A. Hirano, Y. Iijima and T. Ishiguro, "Motion compensated interframe coding for video conferencing", Proc. Nat. Telecommunication Conference. New Orleans, LA, 1981, pp. G5.3.1-G5.3.5.

[18] Li Renxiang, Bing Zeng and Ming L. Liou, "A New Three-Step search algorithm for block motion estimation", IEEE Transactions on Circuits and System for Video Technology, vol. 4, no. 4, 1994, pp. 438-442.

[19] Jianhua Lu and Ming L. Liou, "A Simple and Efficent search algorithem for block matching motion estimation", IEEE Transactions on Circuits and System for Video Technology, vol. 7, no. 2, 1997, pp. 429-433.

[20] Lai-Man Po and Wing-Chaungh Ma, "A Novel FourStep search algorithm for block matching motion estimation", IEEE Transactions on Circuits and System for Video Technology, vol. 6, no. 3, 1996, pp. 313-317.

[21] Shan Zhu and Kai-Kuang Ma, "A New Diamond search for fast block matching motion estimation", IEEE Transactions on Image Processing, vol. 9, no. 2, 2000, pp. 287-290.

[22] You Nie and Kai-Kuang Ma, "Adaptive-Rood Pattern search for fast black matching motion estimation", IEEE Transactions on Image Processing, vol. 11, no. 12, 2002, pp. 1442-1448.

[23] Martin Slanin and Vaclav Ricny, "Estimating PSNR in high definition H.264/AVC video sequances using artificial neural networks", IEEE Radioengineeing, vol. 17, no. 3, 2008, pp. 103-108.

[24] Pascal Flewy and Olivier Egger, "Neural network based image coding quality prediction", In Proceeding of the ICASSP 1997, Munich, pp. 3413-3416.

[25] Yong Zhang and Ming Ming Zhang, "Application of artificial neural network in video compression coding", IEEE International Conference on Information Management, Innovation Management and Industrial Engineering 2008, pp. 207-210.

[26] Rishabh Malhotra and Kunio Takaya, "A novel approach for finding the movement of an object in video sequances by an artificial neural network for 2.5D object modeling", In proceeding of the IEEE, CCECE/CCGI, Saskatoon, 2005, pp. 984-987.
[27] A. Gersho and R.M. Gray, "Vector Quantization and Signal Compression", Kluwer Academic Publisher, 1991.

[28] Mohamed Berkane and Patrick Clarysse, "A neural network-based approach to motion estimation with discontinuities", IEEE Proceeding of the Eighth International Conference on Hybrid Intelligent Systems, 2008, pp. 356-361.

[29] Dae-Hyun Ryu, "Block matching algorithm using neural network", IEEE TENCON - Speech and Image Technologies for Computing and Telecommunications, 1997, pp. 379-382.

[30] K. Ramchandran, A. Ortega and M. Vetterli, "Bit allocation for dependent quantization with applications to multiresolution and MPEG video coders", IEEE Transaction Image Processing, vol. 3, no. 5, 1994, pp. 533-545.

[31] Sabir M.F., Heath R.W. and Bovik A.C., "Joint sourcechannel distortion modeling for MPEG-4 video", IEEE Transactions on Image Processing, vol. 18, no. 1, 2009, pp. $90-105$.

[32] Vikas Sagar, Dr. Krishan Kumar, "A Symmetric Key Cryptography using Genetic Algorithm and Error Back Propagation Neural Network" $9^{\text {th }}$ INDIACom; INDIACom-2015 $20152^{\text {nd }}$ International Conference on "Computing for Sustainable Global Development", $11^{\text {th }}$ $13^{\text {th }}$ March, 2015 Bharati Vidyapeeth's Institute of Computer Applications and Management (BVICAM), New Delhi (INDIA).

[33] Vikas Sagar, Dr. Krishan Kumar, “A Symmetric Key Cryptographic Algorithm Using Counter Propagation Network (CPN)" ICTCS '14, November 14 - 16 2014, Udaipur, Rajasthan, India Copyright 2014 ACM.

[34] Sanjeev Kumar, Krishan Kumar and Anand Kumar Pandey. Article: A Comparative Study of Call Admission Control in Mobile Multimedia Networks using Soft Computing. International Journal of Computer Applications 107(16):5-11, December 2014.

[35] Sanjeev Kumar, Krishan Kumar, Pramod Kumar, "Mobility Based Call admission Control and Resource Estmation in Mobile Multimedia Networks using Artificial Neural Networks", IEEE International Conference, NGCT, Petroleum University, Dehradun, 45 September, 2015.

[36] Mohit Mittal and Krishan Kumar. Article: Quality of Services Provisioning in Wireless Sensor Networks using Artificial Neural Network: A Survey. International Journal of Computer Applications 117(5):28-40, May 2015 